\title{
Assessment of VANET multi-hop routing over an experimental platform
}

\author{
José Santa \\ Department of Information and Communication Engineering, \\ University of Murcia, \\ Campus de Espinardo, 30100 Murcia, Spain \\ E-mail: josesanta@um.es \\ *Corresponding author
}

\author{
Manabu Tsukada, Thierry Ernst, Olivier Mehani \\ INRIA Rocquencourt, \\ IMARA Team, \\ Domaine de Voluceau, BP 105, 78153 Le Chesnay Cedex, France \\ E-mail: (Manabu.Tkukada | Thierry.Ernst | Olivier.Mehani)@inria.fr
}

\author{
Antonio F. Gómez-Skarmeta \\ Department of Information and Communication Engineering, \\ University of Murcia, \\ Campus de Espinardo, 30100 Murcia, Spain \\ E-mail: skarmeta@um.es
}

\begin{abstract}
Evaluation of vehicular ad-hoc networks (VANETs) over real environments is still a remaining issue for most researchers. There are some works which carry out performance tests to evaluate the communication channel according to physical and MAC conditions. Only a few works deal with multi-hop experimentation in this field, and practically none tests multihop protocols. In this paper an integral VANET testbed is evaluated, using $802.11 \mathrm{~b}$ and a multi-hop network managed by the Optimized Link State Routing protocol (OLSR). Up to four vehicles are used to study the VANET performance over different traffic environments and different metrics are considered to analyse the results in terms of delay, bandwidth, packet loss and distance between nodes. Furthermore, a deeper analysis is carried out to track the routes followed by packets end to end. Since a routing protocol is used, results differ from traditional one-hop and static-route tests, presenting a more realistic study.
\end{abstract}

Keywords: vehicular communications; VANET; experimental evaluation; ad-hoc networks; multi-hop communications; OLSR.

Reference to this paper should be made as follows: XXX

Biographical notes: José Santa is a researcher at the Department of Information and Communication Engineering at University of Murcia. He received his MSc in Computer Science Engineering and his MSc in Advanced Information and Telematics Technologies in 2004 and 2008, respectively, and his Ph.D in Computer Science in 2009. His research interests include context awareness, vehicular communications and terrestrial navigation.

Manabu Tsukada is a Ph.D student at MINES ParisTech and works as researcher at INRIA. He has studied in Jun Murai laboratory in Keio University from September 2002 to May 2007. He got BS in 2005 and MSc 2007 from Keio University. His research interests are mobility support for next generation Internet (IPv6) and communications for intelligent vehicles.

Thierry Ernst holds a PhD in networking and work at INRIA leading the LARA-COM subgroup and performing research on networking protocols for infrastructure-based and infrastructure-less vehicular communications. His $\mathrm{PhD}$, obtained in 2001 from University Joseph Fourier, about "Network Mobility Support in IPv6", now serves as the foundation of Internet communications in ITS architectures currently under standardization.

Olivier Mehani is a cotutelle Ph.D student at Mines ParisTech and University of New South Wales. Most of his research is currently done in collaboration with research labs at Nicta and INRIA. He obtained an engineering degree in Computer Science at Université de Technologie de Compiègne and an MSc in Complex Adaptive Systems at Chalmers Teckniska Högskola in 2007. His research interests include Ambient Intelligence and Pervasive Networks.

Antonio F. Gomez-Skarmeta is a Professor at the Department of Information and Communications Engineering at University of Murcia. He received the MSc degree in Computer Science from the University of Granada in and the BS and the Ph.D degrees in Computer Science from the University of Murcia. His research includes mobile communications, pervasive systems and network security. He has published over 70 journal international papers. 


\section{INTRODUCTION}

Vehicular networks are becoming essential for telematic services inside the Intelligent Transportation Systems (ITS) field. Apart from autonomous solutions, such entertainment and some collision avoidance systems exclusively based on vehicle sensors, for example, distributed and collaborative services extend the driver perception and integrate the vehicle in the traffic environment. Safety services are the most studied ITS solutions in the current literature, where vehicular networks are commonly used to exchange navigation and road-side events with the aim of detecting potential hazards. Nonetheless, comfort, traffic management, and monitoring systems, are also more and more dependent on vehicular networks, and implementations of services such as platooning, vehicle tracking, parking reservation and distributed games are only some examples of this expansion.

There are several communication paradigms involved in vehicular networks, which can be essentially summarised as vehicle to vehicle (V2V), vehicle to infrastructure (V2I), infrastructure to vehicle (I2V), and even infrastructure to infrastructure (I2I). Wireless technologies through 802.11 and cellular networks are the most extended communication links, although their usage depends on concrete applications. Among all possible combinations of communication paradigms and wireless technologies, there is one field specially studied by the ITS community, known as Vehicular Ad-hoc Networks, or VANET. In this case, wireless local area networks, such as 802.11 and DSRC (Dedicated Short Range Communications), are applied into the V2V case using concepts inherited from MANETs (Mobile Adhoc Networks).

Although there are a lot of works related to VANET applications and basic research at physical, MAC and, overall, network layers, there is an important lack of real evaluation analysis. Many VANET solutions and protocols could be considered as non practical designs if they were tested over real scenarios, as it has been proved in MANET [Tschudin et al., 2003]. Performance of VANET protocols based on a pure broadcast approach can be more or less expected in simple configurations, even if they are not experimentally tested; but the number of issues concerning the real performance of multi-hop designs is much more tricky. As we detail in next section, the amount of works related with real evaluation of VANET designs is limited, rare if we consider the concrete case of multi-hop transmissions, and practically null in the evaluation of routing protocols. Performing real evaluations in VANET research imply a number of issues, most of them inherited from MANET, and even accentuated. Some of the most important drawbacks of performing VANET experiments are:

- Implementation of routing protocols and/or applications. Most VANET projects end at the simulation stage; hence, almost none of them implement the real

Copyright (C) 200x Inderscience Enterprises Ltd. system.

- Equipment cost. The hardware cost which imply the evaluation of a VANET architecture might not be assumed by all researching groups. If a multi-hop solution is considered, a minimum of three cars equipped with some kind of computer and communication interfaces are necessary.

- Logistic issues in experiments. Not only the necessary equipment is important, but also people to drive cars and control the nodes is essential. When the number of vehicles grow, setting-up experiments is more and more difficult.

- Necessary work to cover a meaningful set of tests. When nodes are ready, a number of realistic scenarios have to taken into account to get meaningful results, what can be a time-consuming task.

In this paper, a multi-hop VANET is evaluated over real environments, setting-up four cars with the necessary equipment, and involving an interdisciplinary group of people which works on ITS. The goal of the work is becoming a reference point for the VANET community, giving an evaluation of a real multi-hop VANET and IPv6 platform, which uses a standardised ad-hoc routing protocol, as the Optimised Link State Routing (OLSR) protocol is. Testing scenarios have been divided into urban and highway; mobility has been set to static, urban-like speed, and high speed; and a wide range of performance metrics have been used, such as bandwidth, RTT (Round-Trip delay Time), jitter and PDR (Packet Delivery Ratio). Because a low level study has been carried out, it is also possible to count the number of hops and calculate PDR per link in data transmissions. This study is done by a filtering software, which merges information from GPS, dump files and traffic generator traces. Moreover, specific scenarios to test the routing protocol behaviour have been considered. The data traffic considered in tests embraces the requirements of most ITS applications, hence ICMPv6, TCP and UDP transmissions have been analysed.

The structure of the paper has been divided into the following Sections: Section 2 briefly describes previous works related to VANET experimental evaluation; Section 3 gives a small overview of VANET concepts and OLSR; Section 4 presents the experiment set-up, in terms of hardware used, place of tests, considered scenarios, and the software developed to analyse the network traffic; the results gathered in the tests are analysed in Section 5 and, finally, the paper is concluded in Section 6.

\section{STATE OF THE ART}

Because of the drawbacks described above, literature in experimental evaluation of VANET architectures is limited, although these works are of key importance for the ITS community. Up to now, there are several works dealing 
with this issue, although most of them are still focused on studying the feasibility of 802.11 and DSRC technologies in the vehicular field. In [González et al., 2008] the applicability of $802.11 \mathrm{~b}$ in $\mathrm{V} 2 \mathrm{~V}$ communications is evaluated over urban and highway scenarios, and it is demonstrated that direct line of sight is one of the most important issues in the network performance. The hardware platform considered is similar to the one chosen in the current work, based on an embedded $\mathrm{PC}$ which acts as access router for in-vehicle devices. A similar analysis is found in [Jerbi et al., 2007a], where several evaluation metrics are used to quantify the performance of the wireless channel between two vehicles. Also using 802.11b, in [Singh et al., 2002] authors demonstrate how mobility and environment factors can seriously degrade the network operation. In addition to consider the most common performance metrics in VANET evaluation, the post-processing software described in next sections, implemented to analyse data logs, enable us to trace packets among communication nodes. Using such feature, it is possible to detect link failures and study the routing protocol performance, in terms of number of hops in transmissions.

Communication between a vehicle and a static terminal is also important for some ITS services. This communication pattern can be considered as a subset of I2V and V2I, where vehicles connect with a local end-point installed at the road side. This is the reason why they are called roadside to vehicle and vehicle to road-side communications, RVC and VRC, respectively. VANET evaluation papers, as the current one, usually consider this special case in testing scenarios. In [Wewetzer et al., 2007] a communication scenario considering a static terminal and a moving vehicle is studied in detail. Among all metrics considered in this work, the transmission power is the more original one, determining the maximum communication range. The type of data traffic used to test the performance of the communication channel is also of interest. Most VANET designs use UDP packets, due to poor TCP performance over wireless channels. In [Hui and Mohapatra, 2005] and [Festag et al., 2004] this issue is studied through different configurations; idea also included in the current paper.

The previous works only consider two terminals in performance tests, what is not too representative in VANET research. In [Maltz and Broch, 2001] a MANET is evaluated using up to eight vehicles and a wide set of experiments are performed, however, the testing environment is too small to be significant for the vehicular community. Two recent works evaluate a multi-hop VANET over real conditions, using three [Jerbi et al., 2007b] and even six vehicles [Jerbi and Senouci, 2008]. These papers offer a wide study about a real VANET set-up, and the last one includes an interesting analysis describing the impact of the number of hops on the final performance, what is also treated in the current paper. Nonetheless, static routes are used in that work, presenting a non-realistic vehicular network. Our work, by contrast, considers a real and standardised ad-hoc routing protocol to dynamically modify communication paths. The hardware test-bed presented is also suited for future ITS research, with a flexible in-vehicle and inter-vehicle IPv6 network based on mobile routers.

\section{VEHICULAR AD-HOC NETWORKS}

The origin of VANET is found in the more general researching subject of ad-hoc networks. However, as it is explained later, vehicular networks present specific conditions which have to be specially studied. OLSR is a well-known proposal of an ad-hoc routing protocol, whose performance in a VANET environment is analysed in this paper.

\subsection{Mobile Ad-hoc Networks}

Mobile Ad-hoc Networks (MANET) are designed to enable wireless communications in dynamic topologies without any infrastructure. In order to adapt to topology changes, MANET nodes exchange control messages to establish the routes used to forward data packets. MANETs have the additional advantage of extending the one-hop communication range, since the packets can be delivered through multiple nodes. MANET routing protocols [Chang et al., 2005] can be classified into the proactive ones, where nodes periodically exchange messages to create routes, and the reactive protocols, in which control messages are exchanged on demand when it is necessary to reach a terminal. Generally, proactive protocols have the advantage of starting communication rapidly by making the routing table ahead; however, this makes battery life shorter due to frequent signalling. If the topology is highly dynamic and the data traffic is frequent, a proactive protocol could be more appropriate. Reactive protocols, on the contrary, keeps the battery life longer by reducing signalling messages when there is no data to transmit.

Some routing protocols specified by the IETF MANET working group ${ }^{1}$ are: the proactive Optimized Link State Routing (OLSR) [Clausen and Jaquet, 2003] and the Topology Dissemination Based on Reverse-Path Forwarding (TBRPF) [Templin and Lewis, 2004]; and the reactive Ad hoc On-Demand Distance Vector Routing (AODV) [Perkins et al., 2003], the Dynamic Source Routing (DSR) [Johnson et al., 2007] and the Dynamic MANET Ondemand (DYMO) [Chakeres and Perkins, 2008].

\subsection{MANET vs. VANET}

Vehicular Ad-hoc Networks (VANET) [Li and Wang, 2007] are a particular case of MANET, which are characterised by battery constraints free, high speed and regular distribution and movement. First, vehicles have a better battery than mobile terminals or sensor devices, which is also charged when the engine is on. Second, the speed of vehicles is also higher than common portable terminals, and relative speeds can reach $300 \mathrm{Km} / \mathrm{h}$; hence, the duration of the routing entries is extremely short. Third, the movement and

\footnotetext{
${ }^{1}$ http://www.ietf.org/html.charters/manet-charter.html
} 
density of the nodes are not random, since vehicles drive on roads, what makes the nodes position somehow predictive. This concept can be used to detect stable structures or clusters to improve the network performance [Little and Agarwal, 2005]. Moreover, a GPS device can be assumed in many cases, whose information improves the network performance in some proposals [Maihöfer, 2004, Mauve et al., 2001, Sun et al., 2006]. In the same way, it would be also possible to send packets only to a set of nodes grouped in a geographical area (geocast) [Liao et al., 2000].

\subsection{OLSR}

OLSR is the routing protocol used in the experiments performed in the work, as it has been stated. Control overhead of this proactive protocol is quite reduced by multipoint relays (MPRs), which characterise OLSR. Each node selects its MPRs among all one-hop terminals, assuring that all neighbouring nodes at two hops can be reached through a minimum set of them. By using MPRs, the network overhead decreases when node density is high, since only the nodes designated as MPRs forward messages. OLSR nodes detect each other by HELLO messages, which are periodically sent. Topology Control (TC) messages are used to disseminate neighbour information throughout the network. Since OLSR nodes can interconnect different networks, another special message is also periodically disseminated to advertise these in the OLSR network, called Host and Network Association (HNA) message. In VANETs, this information is necessary, for example, to exchange invehicle network addresses. This is the case of the communication platform presented in the paper, where each on-board unit acts as a mobile router (MR) for in-vehicle devices.

OLSR is a well-known protocol in the MANET literature. Since the application of MANET concepts in the particular VANET case is a common procedure, the results given in this paper assess how a common ad-hoc proactive protocol operates under vehicular conditions. Because vehicles are not constrained by battery restrictions, one may think that a proactive protocol tuned for highly dynamic topologies could be suitable in the vehicular domain. Evaluating this idea is a key point in the work; hence, the VANET testbed presented in next section uses the OLSR protocol.

\section{EXPERIMENT SET-UP}

A set of common vehicles have been equipped with the necessary hardware to create a VANET using OLSR. Network traffic and positioning information is logged and then processed in order to analyse the VANET performance. The experiment set-up is described in detail in this section, in terms of hardware and software modules developed, network metrics considered and traffic scenarios.

\subsection{Testbed Platform}

Up to four Citröen C3 cars have been used in the trials, mounting the proper hardware to integrate the vehicle in the VANET and log positioning and network traffic information. Figure 1 illustrates the main components of the on-board platform, during one of the field trials. As can be seen, an embedded computer is used as mobile router (MR) in each car. This comprises a Soekris net4521, with a mini-PCI 802.11 Texas Instruments ACX 111802.11 b/g wireless transceiver and a compact flash hard disk. The wireless interface has been set-up at $11 \mathrm{Mbps}$, emulating an 802.11 b device. The computer is connected, via serial port, with a Trimble AgGPS 323 GPS receiver, whose external antenna is visible in the photo. The wireless card uses another external antenna, fixed on the car's roof too. One of the two ethernet connections of the MR is used to connect it with the in-vehicle wired network, by means of a hub. In the sender and receiver vehicles, a laptop is connected to the in-vehicle network. The sender laptop is a Windows XP-based system, whereas the second one comprises a Linux Debian computer.

A Linux Voyage distribution with kernel 2.6.22 has been installed on MRs, and the olsr.org daemon 0.5.6-rc7 $7^{2}$ (an implementation of the OLSR protocol) has been configured on each one. The OLSR configuration parameters are listed in Table 1 . The transmission period of hello packets has been adjusted to deal with vehicle mobility, through a set of preliminary tests and considering a previous study about tuning OLSR in movility environments [Cheng et al., 2006]. Since the topology is highly variable, the same has been made with TC_Interval and TC_ValidityTime periods. It is important to remember that TC messages are useful to exchange topology information between network nodes. MRs do not use more than one interface in the VANET, hence multiple interface declaration (MID) parameters are left with default values. Host and network association (HNA) parameters have been adjusted. Since HNA messages are used to announce other networks accessible through OLSR nodes, increasing the frequency of these notifications makes faster advertising the in-vehicle networks. The last two parameters should have the same value than the topology control ones [Clausen and Jaquet, 2003]. As it has been stated, the network prefixes of in-vehicle networks are advertised using HNA messages of OLSR. These networks are listed in the configuration file of OLSR daemon. This mechanism implies a set of security issues, such a denial of service, which should be covered in a real deployment of the system.

\subsection{Data Gathering and Post-Processing Fusion}

An overview of the experimental evaluation process carried out in the work is given in Figure 2. In the tests, up to four vehicles have been used, however, the system is

\footnotetext{
${ }^{2}$ http://www.olsr.org/
} 
Table 1: OLSR configuration parameters

\begin{tabular}{|c|c|c|}
\hline Parameter & Value (s) & Default (s) \\
\hline Hello_Interval & 0.5 & 2.0 \\
Hello_Validity_Time & 6.0 & 6.0 \\
TC_Interval & 3.0 & 5.0 \\
TC_Validity_Time & 9.0 & 15.0 \\
MID_Interval & 5.0 & 5.0 \\
MID_Validity_Time & 15.0 & 15.0 \\
HNA_Interval & 3.0 & 5.0 \\
HNA_Validity_Time & 9.0 & 15.0 \\
\hline
\end{tabular}

prepared to consider any number of vehicles. The sender laptop is in charge of generating data traffic, and both the sender and the receiver save a high level log, according to the application used to generate network traffic. All MRs save information about forwarded data packets, by means of the tcpdump software ${ }^{3}$, and log the vehicle position continuously. All these data is analysed in post-process by the AnaVANET software. This is a Java application which traces all the data packets transmitted from the sender node. This way, it is possible to detect packet losses and calculate statistics for each link and end-to-end, and merge all these per-hop information with transport level statistics of the traffic generator. As a result, AnaVANET outputs an XML file with statistics of one-second periods, and a packet trace file with the path followed by each data packet. The first file is uploaded to a Web server, which uses Google Maps functionalities to graphically replay the tests. The Graphic Generator module gives another view of the network performance, using both XML and packet traces to process results and then create several types of figures through the GNU Plot utility.

\subsection{Analysed Traffic and Performance Metrics}

Three different types of data traffic have been considered in the tests carried out over the IPv6 network. These are described in next points, together with the software used to generate the packets:

- UDP. A unidirectional transmission of UDP packets from the sender laptop to the receiver one, has been generated using the IPerf tool ${ }^{4}$. The packet length has been fixed to 1450 bytes, to avoid fragmentation with IPv6, and they are sent at a rate of 1 Mbps. Note that the maximum packet length could be fixed at 1452 , since the IPv6 and UDP headers imply 40 and 8 bytes, respectively.

- TCP. A TCP connection is established between the sender and receiver laptops, non limiting the maximum bandwidth. The IPerf tool is again used in the traffic generation and the maximum segment size used

\footnotetext{
${ }^{3}$ http://www.tcpdump.org/

${ }^{4}$ http://dast.nlanr.net/Projects/Iperf/
}

was 1440 , since the IPv6 and TCP headers imply 40 and 20 bytes, respectively.

- ICMPv6. The Windows XP Ping6 utility is used to generate IPv6 ICMP (Internet Control Message Protocol) echo request packets from the sender laptop, and to receive echo reply packets from the remote one.

These three types of traffic have been used to analyse the network performance hop-by-hop and end-to-end, considering the most extended metrics in MANET evaluation [Chang et al., 2005]. In the TCP case, the high level information given by IPerf, at a 0.5 -second rate, is considered in the process. ICMPv6 and UDP packets are, however, traced across nodes. Since there is no fragmentation for UDP packets, a direct correspondence exists between MAC and IP layer packets in the study. At this level, the packet delivery ratio (PDR), the number of hops and the jitter are calculated. For ICMPv6 data flows, the round-trip delay time (RTT) have been also considered. At the transport level, the IPerf information used is the instantaneous bandwidth.

\subsection{Experimental Scenarios}

A set of scenarios have been considered with the aim of obtaining significant results under different conditions. The main factors which determine these scenarios are:

- Mobility. Static and dynamic scenarios have been considered to test the network operation under controlled and common traffic settings.

- Environment. Two different environments have been considered: a semi-urban one, inside INRIARocquencourt installations, which contains a set of small buildings surrounded by streets, and a highway stretch, the French A-12, near INRIA-Rocquencourt.

- Number of vehicles. Up to four vehicles are considered in the field trials, in order to check the delay increase with the number of hops.

The various traffic types (UDP, TCP and ICMPv6) have been applied over each defined scenario. In Figure 3, four common scenarios in VANET evaluation, with up to three vehicles, are illustrated. The first one has been used to check the maximum communication range between two vehicles, with the aim of isolating the creation and loss of one link in the OLSR network. The second scenario considers a typical urban environment, where a building (or a set of them) hides the line of sight between the source and the destination cars (blue and black, respectively). A multihop network is suited in this kind of situations. A new vehicle (number two, in red color) forwards the messages received from the source vehicle to the destination one. In the third scenario the three vehicles move around this blockage area. The last scenario of Figure 3 (number four) uses the same vehicles over a highway environment. 
Figure 4 shows the overtaking and four-vehicle scenarios. The fifth scenario considers static tests with four vehicles, where packets are always forwarded by two intermediate cars. A new car (the fourth one, in green) has been included. In the sixth scenario, an overtaking situation is tested, where the receiver car (number one, in black) and car two (in red) are parked, and the sender one (number three, in blue) passes them in the two directions two times. When the distance between the sender and receiver cars is too big, the direct link is lost, and the OLSR protocol setsup a multi-hop path using car two (in red) as a relay node. The sender car, after passing the last car in one direction, usually reaches a position where the communication is lost. The last scenario follows the same idea, but four vehicles are now used, using car four (in green) as another relay node. The parked vehicles are more separated now, to avoid non-desired direct links.

\section{EVALUATION AND ANALYSIS}

The VANET experiments described in the previous seven scenarios have been performed considering the UDP, TCP and ICMPv6 traffic types. Results of the experiments have been processed by AnaVANET and then showed with a graphical software. Multitude of plots have been generated and used in this section to analyse the main results of the vast experimental evaluation.

\subsection{Web-Based Network Analysis}

The carried out experiments are available in our public website $^{5}$, and they can be replayed to see the momentary performance of the network during the tests. Figure 5 shows a screenshot of the website. All the experiments can be selected and main performance metrics can be monitored at any time. Users can play and stop at any arbitrary point of the test with the control bottons on the left side of the page. The player speed, one step forward and one step backward are also implemented. On the map, the position and movement of the vehicle are depicted with the speed of each vehicle and the distance between them. The transferred data size, bandwidth, packet loss rate, roundtrip delay time and jitter, for each link and end to end are displayed. The user can see the network performance by attending the width of link lines and the colour used to draw them.

\subsection{Maximum Range Tests}

Maximum range tests have been performed with two cars through Scenario 1. The sender starts leaving from the receiver vehicle position (static), and then it comes back, at about 180 meters, to approach again to the initial point. The speed of the sender was maintained under $10 \mathrm{Km} / \mathrm{h}$ to smoothly check the loss of connectivity.

\footnotetext{
${ }^{5}$ http://fylvestre.inria.fr/ $\sim$ tsukada/experiments/vanet-jose/
}

Figure 6 shows PDR in the case of the UDP transmission. Packets start to be dropped around 100 meters of distance. The last packet arrives around 120 meters away and, after this point, there are no delivered packets, until the sender vehicle comes back and reaches 100 meters of distance. Since periodical OLSR control messages are lost when the distance is around 120 meters, the path is removed of the routing table and the transmission ends at this point. The jitter in the same test is illustrated in Figure 7 . When the sender car leaves the receiver one, at a distance between 75 and 120 meters, the jitter is higher, due to layer-two retransmissions caused by the increase of the distance. When the sender approaches the receiver again, this effect is again visible at distances between 100 and 50 meters. It is noticeable how the communication is lost at a point further away than it comes back. This is due to timeout periods in the reception of control messages give an extra time to maintain the communication link. When the vehicle comes back, some signalling traffic must be also exchanged before the routing table of the sender vehicle is updated.

The TCP performance over the same scenario is showed in Figure 8. As can be seen, only a one-way path has been logged. When the route is lost at 100 meters of distance, the TCP timeout expires and the transport layer link is broken. Finally, Figure 9 shows the RTT values collected in a Ping test over the same scenario, measured end to end. The base line of RTT is about $10 \mathrm{~ms}$, but several peaks appear even under good conditions, due to route updates carried out by OLSR and the movement of the sender vehicle. The communication is again lost at a similar distance to the previous cases, however, it comes back earlier than in the UDP test. This is due to the network overhead is much lower in the Ping test (only one message per second), hence the OLSR signalling messages can be efficiently sent and the communication is reestablished earlier.

\subsection{Static Tests}

Static results using Scenarios 2 and 5 are summarised in Table 2, using three and four vehicles, respectively. The duration of all the tests was 15 minutes. The total distance between the sender and receiver cars was 120 meters $(70$ plus 50 meters) in Scenario 2, and 155 meters (50 plus 70 plus 35 meters) in Scenario 5. As can be seen, the UDP performance is almost ideal, considering that the emission of packets was fixed at a rate of $1 \mathrm{Mbps}$. Packet losses are not frequent, and the mean PDR is $99.99 \%$. Small variances of performance are only due to route updates, noticeable in jitter values. In TCP results, the average bandwidth is $1.9 \mathrm{Mbps}$, what reveals a good performance too. However, frequent variations are evident if the standard deviation (STD) is considered. Since the vehicles are static and the network topology does not present variations, wireless conditions are considered to impact on the TCP performance. By means of the slow start mechanism, TCP dynamically adjusts the transmission rate accord- 
Table 2: Network performance in static tests

\begin{tabular}{|c|l|r|r|r|r|}
\hline Test & Metric & Min. & Ave. & Max. & STD \\
\hline \hline $\begin{array}{c}\text { UDP } \\
\text { 3 v. }\end{array}$ & $\begin{array}{l}\text { PDR } \\
(\%)\end{array}$ & 98.84 & 99.99 & 100 & 0.11 \\
\cline { 2 - 6 } & $\begin{array}{l}\text { Band. } \\
\text { (Kbps) }\end{array}$ & 545.20 & 1001.59 & 1020.8 & 34.15 \\
\cline { 2 - 6 } & $\begin{array}{l}\text { Jitter } \\
\text { (ms) }\end{array}$ & 0.14 & 0.57 & 5.57 & 0.78 \\
\hline $\begin{array}{c}\text { TCP } \\
\text { 3 v. }\end{array}$ & $\begin{array}{l}\text { Band. } \\
\text { (Kbps) }\end{array}$ & 327.68 & 1915.95 & 2282.24 & 359.1 \\
\hline $\begin{array}{c}\text { Ping } \\
\text { 3 v. }\end{array}$ & $\begin{array}{l}\text { RTT } \\
\text { (ms) }\end{array}$ & 4.00 & 4.96 & 23 & 1.38 \\
\hline $\begin{array}{c}\text { Ping } \\
\text { 4 v. }\end{array}$ & $\begin{array}{l}\text { RTT } \\
(\mathrm{ms})\end{array}$ & 6.00 & 7.25 & 19 & 1.49 \\
\hline
\end{tabular}

ing to network performance, but this algorithm does not converge properly in wireless networks. Frequent packet losses, mainly due to interferences in the medium, and the presence of eventual route updates imply an unstable network environment for TCP. During all static tests, the wireless channel was chosen among all of them not used in the surroundings at the beginning of the trial, but interferences in the medium could not be avoided at all.

Ping tests show the good two-way latency of the network. With three vehicles, the average RTT is $4.96 \mathrm{~ms}$, but this value is exceeded when four vehicles are considered, reaching a mean RTT of 7.25. Hence, the addition of one hop increments the latency by more than $2 \mathrm{~ms}$. The RTT standard deviation is also higher in the last case, due to the new node imply additional control traffic and, overall, new occasional route updates. Moreover, since the route from the source to the destination terminals comprises a linear path across the four MRs, as the number of nodes increases, the probability of finding routing or delay problems along the path is higher.

\subsection{Dynamic Tests under Urban Condi- tions}

According to Scenario 3, three vehicles have been driven around a set of buildings, with the intention of blocking the direct link between Cars 3 and 1 . The speed of the test where set between $15 \mathrm{~km} / \mathrm{h}$ and $30 \mathrm{~km} / \mathrm{h}$. The right and left roads illustrated in Scenario 3 are in reality very narrow, hence some communications problems appear in the corners.

The results collected in the UDP test are plotted in Figure 10. The upper plot shows the number of hops used in the paths followed by UDP packets, whereas the lower graphs show the PDR, computed end to end and per link. PDR is calculated per second, while the number of hops is plotted for each packet transmitted from the sender node. When no hops are drawn, the route to the destination vehicle is not available. Zero hops means that the packet was sent by the first MR, but it was not received by any other. Negative values represent those packets which did not arrive to the destination vehicle, but some hops were reached. As can be seen, a direct relation exists between PDR and number of hops. When this last value is equal or lower than zero, the PDR decreases. When the vehicles are in the same street, some direct paths (one-hop) appear; however, when the distance between the sender and the receiver cars is large enough, the two-hop route is used. These different types of paths can be also seen if the perlink PDR is observed. Whereas the direct link (MR3-MR1) gives intermediate PDR values, the PDR between consecutive vehicles is almost identical and near $100 \%$ when the two-hop link is used, due to the lower distance between nodes.

Since communication problems appear at corners in some laps, OLSR signalling messages are lost, and the communication is temporary down. An interesting effect is also noticeable at time 250 seconds of the UDP test. Here, several paths with more than two hops are registered. This is due to routing problems of OLSR when the destination node suddenly disappear, what provokes cycles in the network. When the network topology is finally updated in all the nodes, these packets are finally dropped, if the receiver node is out of range, or they reach the destination. These cycles provoke a great eventual jitter, which is also highly variable in the rest of the test, due to stressful conditions.

The bandwidth obtained in the TCP test is showed in Figure 11. The performance of the network is very good in the first fifty seconds, due to the vehicles started the trial parked very near. However, the rest of the test shows a high variability, due to continuous changes in topology and communication problems in corners. When conditions are favourable, TCP tries to normalise the bandwith, but soon a link disappears and the bandwidth falls. Peaks of performance are obtained when the sender and receiver cars are in a direct line of sight. TCP timeouts do not expire because there are no long disconnection periods, hence the transport-level communication is maintained.

The final test (Figure 12) comprises a Ping transmission. As can be seen, several steps appear between two main RTT values: five and seven milliseconds. This match with two-hop and four-hop two-way paths. Several three-hop routes have been collected, due to, sometimes, the ICMP Echo Request packets take a different route than the Echo Reply ones. If the ratio of non delivered packets (negative hop counts in this case) is compared with the one obtained in the UDP test, it is noticeable how it is lower now. Since the data traffic is much more lower in the Ping case (one message per second), signalling traffic is more efficiently propagated, and changes in network topology are earlier known.

\subsection{Dynamic Tests in Highway}

The dynamic tests performed over highway conditions follow Scenario 4. The speed of the cars was around 100 $\mathrm{km} / \mathrm{h}$, but the distance between vehicles was variable, due to the rest of traffic on the road. Moreover, communica- 
tion problems in this test are not only due to buildings, but also to surrounding vehicles.

The PDR obtained in the UDP test is presented in the lower part of Figure 13. As can be seen, when the distance between vehicles increases, the PDR becomes lower. As in the urban scenario, intermediate values between 0 and $100 \%$ are not very frequent, due to OLSR remove the routes between nodes when signaling packets are lost. At the beginning, the network performance is good, due to the direct path is chosen, as can be seen in the partial PDR study of the MR3-MR1 link. When vehicles start to separate, the two-hop path is used, as it is showed in the PDR of MR2-MR1 and MR3-MR2 links and the number of hops of chosen paths, showed in the upper graph. High variations of distance provoke route updates and, therefore, packet losses. Around 300 seconds of test, vehicles regroup, but the three-hop path is maintained, due to the time OLSR needs to adapt to the new topology. The high variability of distance around time 350 seconds, makes the network does not stabilise and many packets are lost. A higher period of $0 \%$ of PDR is noticeable, however, around 150 seconds of test. In this case, the communication between the sender vehicle and the others is blocked by a near building.

The bandwidth results of the TCP test are showed in Figure 14. The vehicles are grouped at the beginning of the tests and the bandwidth is around $5 \mathrm{Mbps}$. However, when Car 3 enters the highway and the distance with the other two cars increases, the bandwidth dramatically falls. As can be seen during the whole test, there is again a direct relation between the distance of vehicles and the final performance. Taking into account the maximum range and static tests, it is easy to identify in the graph the moments in which a three-hop path is used. Bandwidths around 2 Mbps represent these cases, whereas results between 4 and 5 Mbps belong to direct paths.

Finally, Figures 15-16 show the results collected during the Ping test. As can be seen, the RTT increases when the vehicles are far enough to use a four-hop two-way route. At this moment, the RTT passes from around three milliseconds to reach the five milliseconds. It is advisable again, how intermediate RTT values are not frequent, being the number of hops the main factor which determines the result. When the distance among vehicles grows and communication starts to be difficult, the links between MRs break, due to losses of OLSR signalling messages.

\subsection{Overtaking Tests}

The overtaking tests using three and four vehicles have been carried out according to Scenarios 6 and 7. Two and three cars, respectively, were parked at a straight avenue, to simulate they are driving at the same speed, and the sender vehicle overtakes them several times in both directions (see Figure 4).

The results of the UDP test with three vehicles are depicted in Figure 17. The distances are calculated taking into account the moving vehicle (Car 3 ) as the reference. It can be seen that two-hop routes appear when the sender vehicle is around the non receiver one. However, during periods of route updates, packets are not delivered. Since the parked vehicles are in an open area and they have a direct line of sight, there are no practically packet losses in the MR1-MR2 link. It is advisable how the number of correctly delivered packets is greater between peaks of distance, but shifted to the left. This is due to the time required by OLSR to adapt to the new topology. Therefore, the best results are obtained when the routes are maintained for a long time. This is the case when the sender car comes back after "overtaking" the other two, since the appropriate route was established when the sender vehicle passed them. Figure 17 also reflects that a high jitter is maintained during all the test, due to high dynamism of the network. At the beginning, jitter starts to increase when the sender vehicle goes away of its initial position and, during the rest of the test, it is highly variable due to route updates.

The bandwidth results of the TCP test are plotted in Figure 18. As in the previous TCP tests, the two characteristic values around 5 and $2 \mathrm{Mbps}$ are again visible when one-hop and two-hop routes are established, respectively. As can be seen, the direct path is used when the distance between the sender and receiver cars is lower. The distance curves are now a bit different, because the sender vehicle comes back earlier after passing both cars, in order to maintain the TCP session.

Two ping tests with three and four vehicles were made following the same overtaking pattern. Figure 19 shows the results of the first one. RTT fluctuations between three and five milliseconds, depend wether the two or four-hop two-way paths are chosen. Some three-hop paths have been collected, due to the different path taken by some Echo Request and Echo Reply messages. As can be seen, the behaviour of the network is more regular than in the UDP case, and packet losses are mostly due to peaks of distance. This is explained again by the low data traffic, which allows OLSR control messages to be efficiently transmitted. This way, route updates due to topology changes are made faster. In the four-vehicle test of Figure 20, the paths are more varied. However, one-way paths of three hops are not frequent. OLSR bet on maintaining two-hop paths when communication is possible, hence Car 2 is bypassed when it is "overtaken" the first time. In the second passing of this car, some two-way paths of six hops are collected, however. More packet losses have been collected than in the previous Ping test, because the parked vehicles were further separated to avoid direct paths among them.

\section{CONCLUSIONS}

The work presented in this paper offers a complete testbed for VANET evaluation, suited to carry out performance trials over the IPv6 basis, but, overall, it gives an original evaluation of an ad-hoc routing protocol in the vehicle domain. The platform enables the researcher to 
analyse the network performance at low level. Several logs collected from individual mobile routers are post-processed to calculate several performance metrics at link, network and transport level. This way, typical statistics are obtained, such as the packet delivery ratio, round-trip delay time, jitter and bandwidth; but also new performance metrics are offered, such as the number of hops used to deliver a packet, or the per-link PDR, for example.

Up to four vehicles have been set-up to perform multitude of tests in the surroundings of INRIA Ronquencourt. These cover both urban and highway environments, and take into account static and dynamic conditions. Main results of all these field trials have been analysed in the paper. The results show the great performance difference which can be found if a routing protocol is used in VANET evaluation, in comparison to previous experimental analysis available in the literature based on static routes. Although it has been tuned to dynamic conditions, the OLSR protocol shows limitations to efficiently update routing tables under stressful conditions. This effect is more noticeable when the volume of data traffic is high, due to network overload. The maximum communication range between vehicles also presents a different pattern, if we compare the results with the ones obtained in static route configurations. Now, the communication is not possible when some control messages are lost and OLSR timeouts expire. Hence, obtaining medium/low PDR results is less frequent than in static route tests, due to, when a great part of messages start to be lost the routes become directly unavailable.

The TCP operation over real VANET deployments should be specially taken into account, because the lack of routing information for a while can lead to transportlevel disconnection. In all the tests, the line of sight between vehicles has been a key factor to maintain communication links. Moreover, the number of hops has been identified as another key performance factor. An incremental delay between two and three milliseconds per hop has been detected when direct paths between nodes are not used. These cases can be found when the distance between sender and receiver vehicles increase significantly, or when near buildings block the direct communication. However, it is expected that this increment in delay follows a quasiexponential increase when more vehicles are in the communication range, due to interferences. Moreover, according to the experiences with four nodes, it has been checked that OLSR prefers smaller paths when communication is possible. This has been checked when an overtake scenario has been emulated, with a vehicle passing three cars in an open area.

In the future, the data recorded during the set of tests will be further exploited, considering, for instance, the impact of driving speed in the final performance and the increase in signaling traffic when OLSR parameters are tuned for the vehicular environment, as it has been explained. A more VANET-oriented protocol developed at INRIA will be also evaluated through new field trials, using the presented test-bed. This is located inside the geographic- based routing proposals, which are demonstrating to be the correct direction in vehicular network research. New wireless devices compliant with current 801.11 p specifications have also been acquired in order to perform a new set of trials which can be compared with the results obtained in this work. The different traffic priorities available in 802.11p will be evaluated for propagating signaling and data traffic among nodes. At the University of Murcia, ongoing cellular network evaluations will be soon complemented with WiMAX trials in the vehicular frame, thanks to a current project to deploy a WiMAX infrastructure in the Campus of Espinardo.

\section{ACKNOWLEDGMENTS}

The Authors would like to thank the Ministerio de Ciencia e Innovación for sponsoring these research activities under the grant TIN2008-06441-C02-02. This work has been partially carried out inside the Intelligent Systems group of the University of Murcia, awarded as an excellence researching group in frames of the Spanish Plan de Ciencia y Tecnología de la Región de Murcia (04552/GERM/06).

\section{References}

[Chakeres and Perkins, 2008] Chakeres, I. and Perkins, C. (2008). Dynamic MANET On-demand (DYMO) Routing. IETF, draft-ietf-manet-dymo-16.

[Chang et al., 2005] Chang, Z., Gaydadjiev, G., and Vassiliadis, S. (2005). Routing protocols for mobile ad-hoc networks: Current development and evaluations. In $A n$ nual Workshop on Circuits, Systems and Signal Processing, pages 489-494, Veldhoven, Holland.

[Cheng et al., 2006] Cheng, Y., Bhatti, S., and Parker, D. (2006). Tuning OLSR. In IEEE International Symposium on Personal, Indoor and Mobile Radio Communications, pages 1-5, Helsinki, Finland.

[Clausen and Jaquet, 2003] Clausen, T. and Jaquet, P. (2003). Optimized Link State Routing protocol (OLSR). IETF MANET Working Group. RFC 3626.

[Festag et al., 2004] Festag, A., Fubler, H., Hartenstein, H., Sarma, A., and Schmitz, R. (2004). Fleetnet: Bringing car-to-car communication into the real world. In ITS World Congress, Nagoya, Japan.

[González et al., 2008] González, V., Santos, A. L., Pinart, C., and Milagro, F. (2008). Experimental demonstration of the viability of IEEE $802.11 \mathrm{~b}$ based inter-vehicle communications. In TridentCom '08: Proceedings of the 4 th International Conference on Testbeds and research infrastructures for the development of networks $\&$ communities, pages 1-7, Innsbruck, Austria. 
[Hui and Mohapatra, 2005] Hui, F. and Mohapatra, P. (2005). Experimental characterization of multi-hop communications in vehicular ad hoc network. In ACM international workshop on Vehicular ad hoc networks, pages 85-86, Cologne, Germany.

[Jerbi et al., 2007a] Jerbi, M., Marlier, P., and Senouci, S. M. (2007a). Experimental assessment of V2V and I2V communications. In Proc. IEEE Internatonal Conference on Mobile Adhoc and Sensor Systems MASS 200\%, pages 1-6, Pisa, Italy.

[Jerbi and Senouci, 2008] Jerbi, M. and Senouci, S. M. (2008). Characterizing multi-hop communication in vehicular networks. In Proc. IEEE Wireless Communications and Networking Conference WCNC 2008, pages 3309-3313, Las Vegas, USA.

[Jerbi et al., 2007b] Jerbi, M., Senouci, S. M., and Al Haj, M. (2007b). Extensive experimental characterization of communications in vehicular ad hoc networks within different environments. In Proc. VTC2007-Spring Vehicular Technology Conference IEEE 65th, pages 2590-2594, Dublin, Ireland.

[Johnson et al., 2007] Johnson, D., Hu, Y., and Maltz, D. (2007). The Dynamic Source Routing Protocol (DSR) for Mobile Ad Hoc Networks for IPv4. IETF, RFC 4728.

[Li and Wang, 2007] Li, F. and Wang, Y. (2007). Routing in vehicular ad hoc networks: A survey. IEEE Vehicular Technology Magazine, 2(2):12-22.

[Liao et al., 2000] Liao, W.-H., Tseng, Y.-C., Lo, K.-L., and Sheu, J.-P. (2000). GeoGRID: a geocasting protocol for mobile ad hoc networks based on grid. Journal of Internet Technology, 1(2):23-32.

[Little and Agarwal, 2005] Little, T. and Agarwal, A. (2005). An information propagation scheme for VANETs. In 8th International IEEE Conference on Intelligent Transportation Systems, pages 155-160, Vienna, Austria.
[Maihöfer, 2004] Maihöfer, C. (2004). A survey of geocast routing protocols. IEEE Communications Surveys and Tutorials, 6(2):32-42.

[Maltz and Broch, 2001] Maltz, D. and Broch, J.and Johnson, D. (2001). Lessons from a fullscale multihop wireless ad hoc network testbed. IEEE Personal Communications, 8(1):8-15.

[Mauve et al., 2001] Mauve, M., Widmer, J., and Hartenstein, H. (2001). A survey on position-based routing in mobile ad hoc networks. IEEE Network Magazine, 15(6):30-39.

[Perkins et al., 2003] Perkins, C., Belding-Royer, E., and Das, S. (2003). Ad hoc On-Demand Distance Vector (AODV) Routing. IETF, RFC 3561.

[Singh et al., 2002] Singh, J., Bambos, N., Srinivasan, B., and Clawin, D. (2002). Wireless LAN performance under varied stress conditions in vehicular traffic scenarios. In Vehicular Technology Conference Fall, volume 2, pages 743-747, Vancouver, Canada.

[Sun et al., 2006] Sun, W., Yamaguchi, H., Yukimasa, K., and Kusumoto, S. (2006). Gvgrid: A QoS routing protocol for vehicular ad hoc networks. In Proc. 14th IEEE International Workshop on Quality of Service IWQoS 2006, pages 130-139, New Haven, USA.

[Templin and Lewis, 2004] Templin, R. O. F. and Lewis, M. (2004). Topology Dissemination Based on ReversePath Forwarding (TBRPF). IETF, RFC 3584.

[Tschudin et al., 2003] Tschudin, C., Lundgren, H., and Nordstrom, E. (2003). Embedding MANETs in the real world. Lecture notes in computer science, 2775(1):578 589 .

[Wewetzer et al., 2007] Wewetzer, C., Caliskan, M., Meier, K., and Luebke, A. (2007). Experimental evaluation of UMTS and wireless LAN for inter-vehicle communication. In International Conference ITS Telecommunications, pages 287-292, Sophia Antipolis, France. 


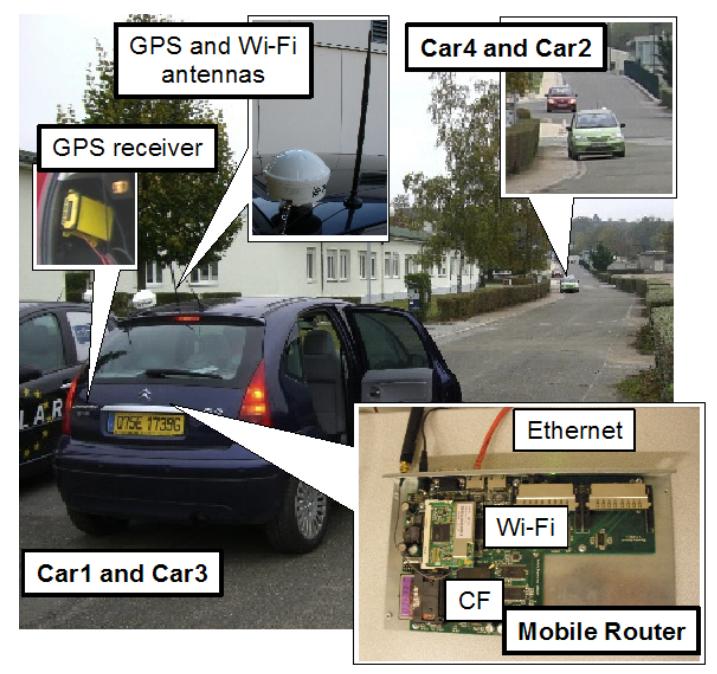

Figure 1: Equipment used in tests.

\section{Experiments}
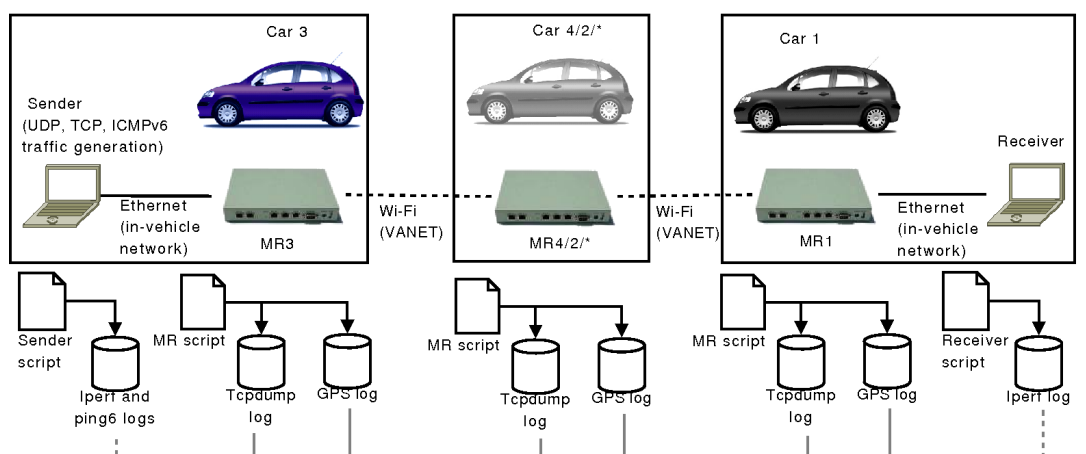

Processing

Analysis
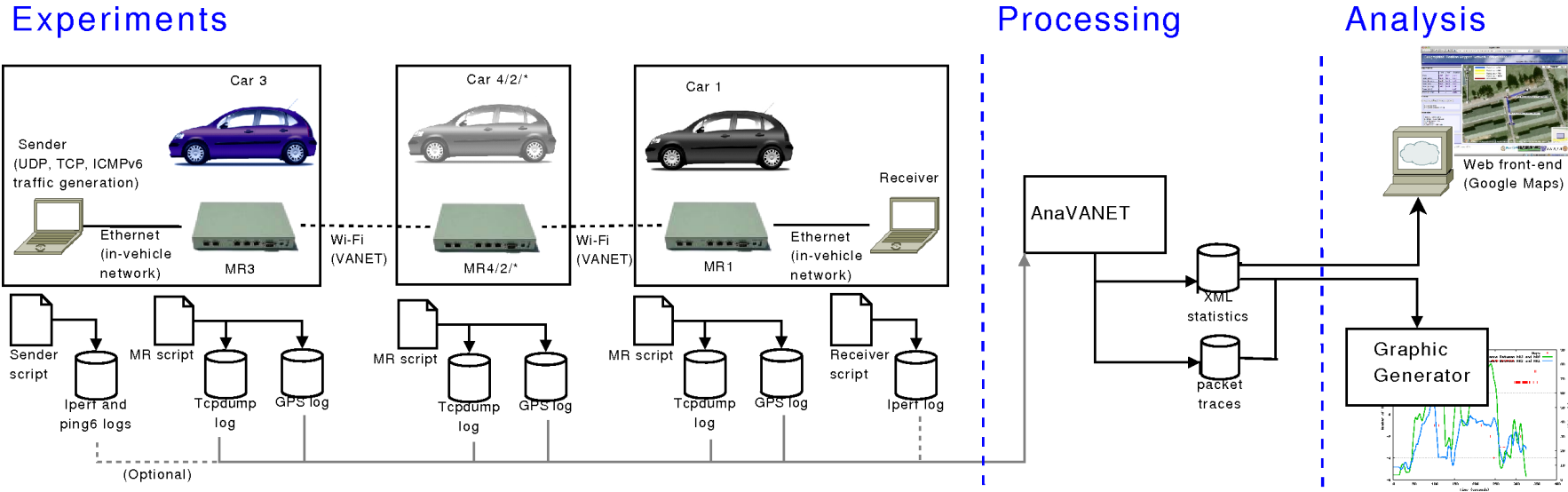

Figure 2: System overview and data processing units.
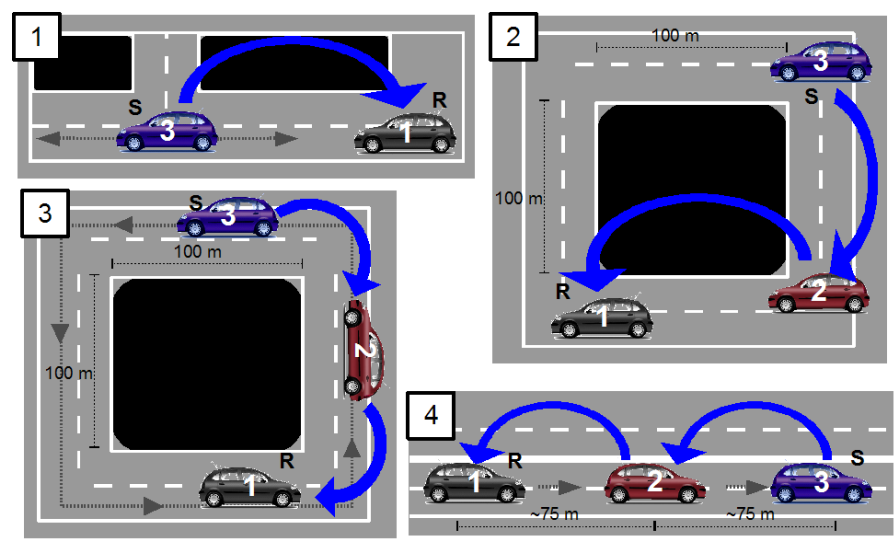

Figure 3: Maximum range and three-vehicles tests. 

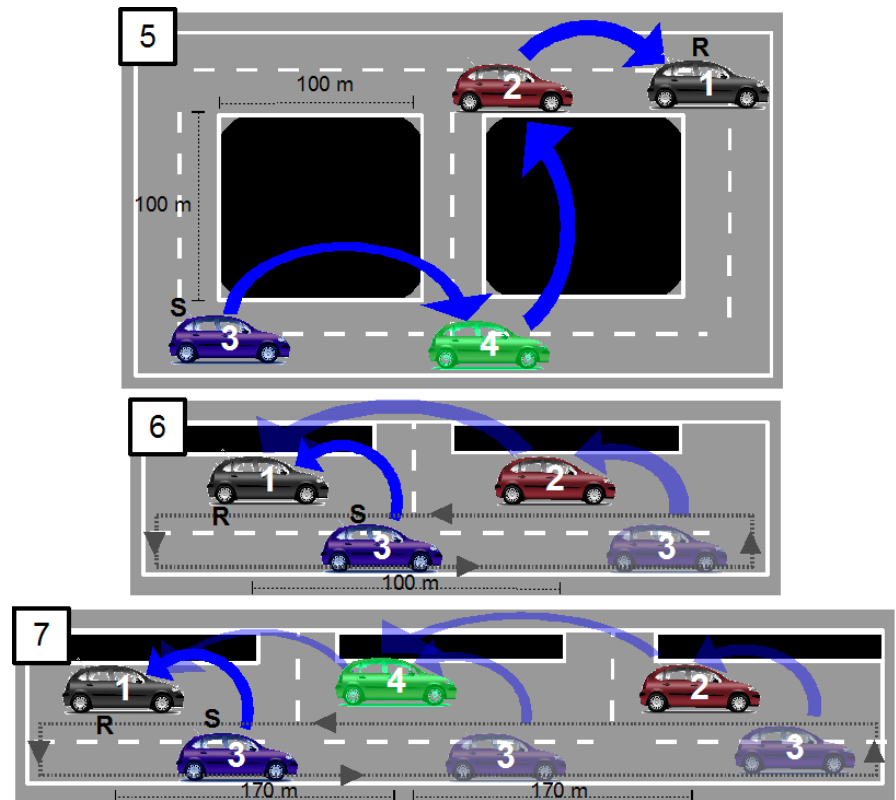

Figure 4: Overtaking and four-vehicles tests.

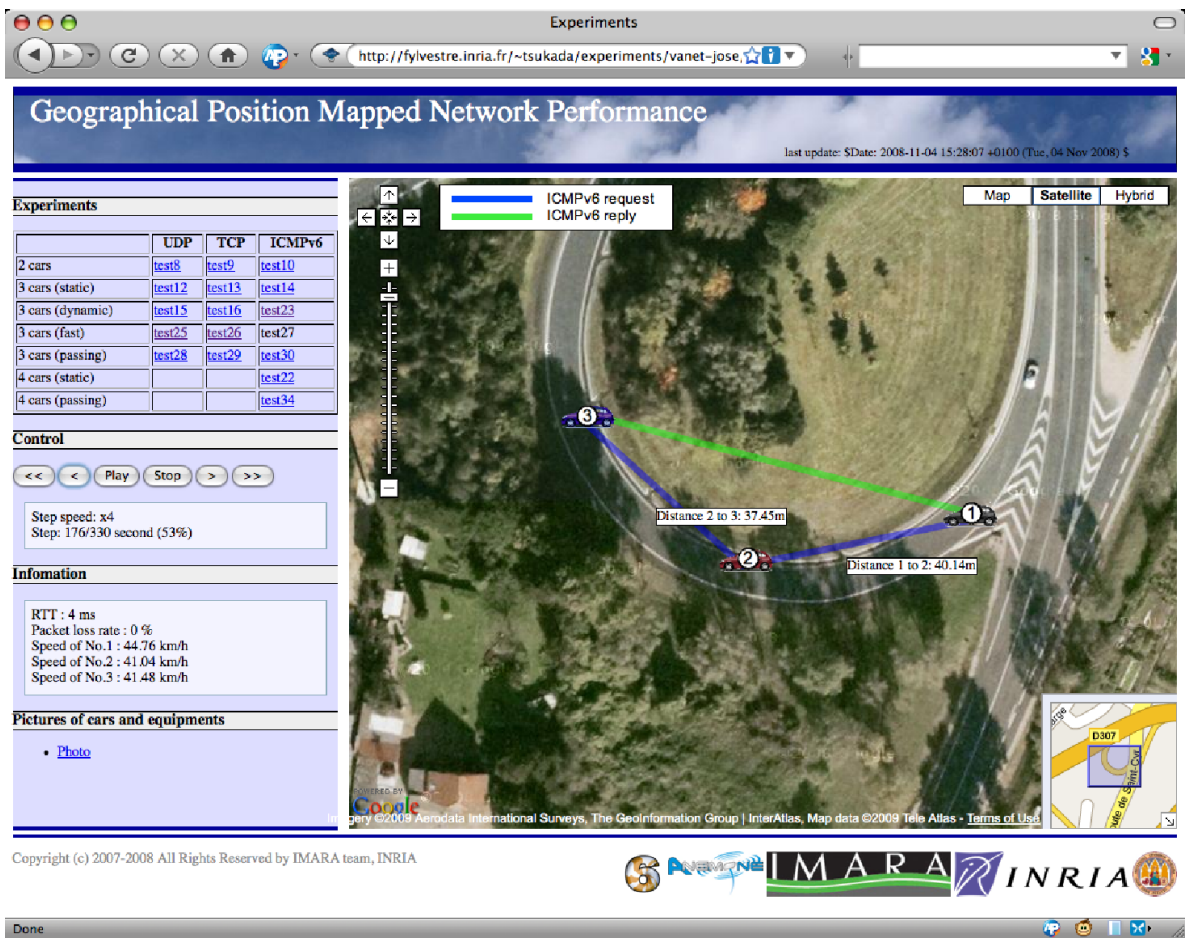

Figure 5: Screenshot of the Report Website. 


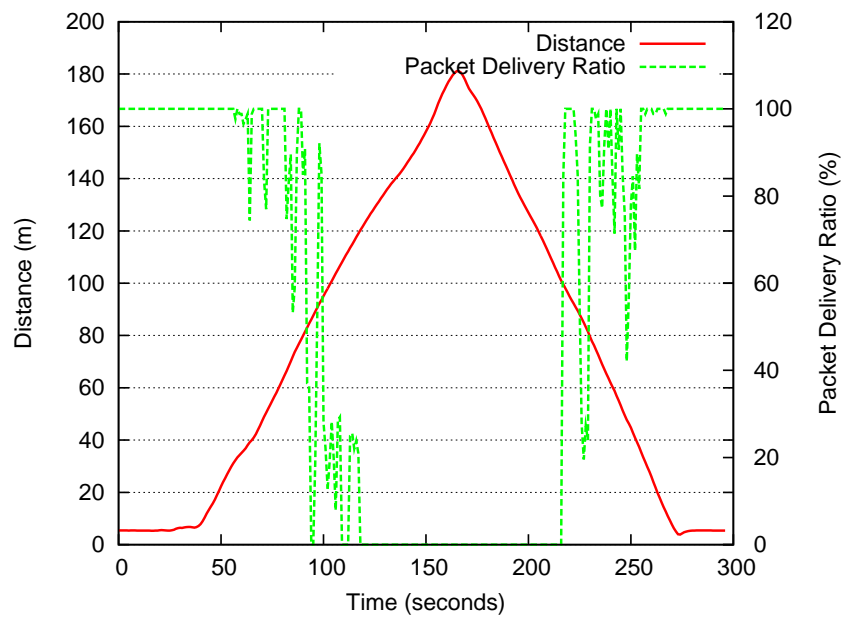

Figure 6: UDP range test with 2 cars (dist./PDR).

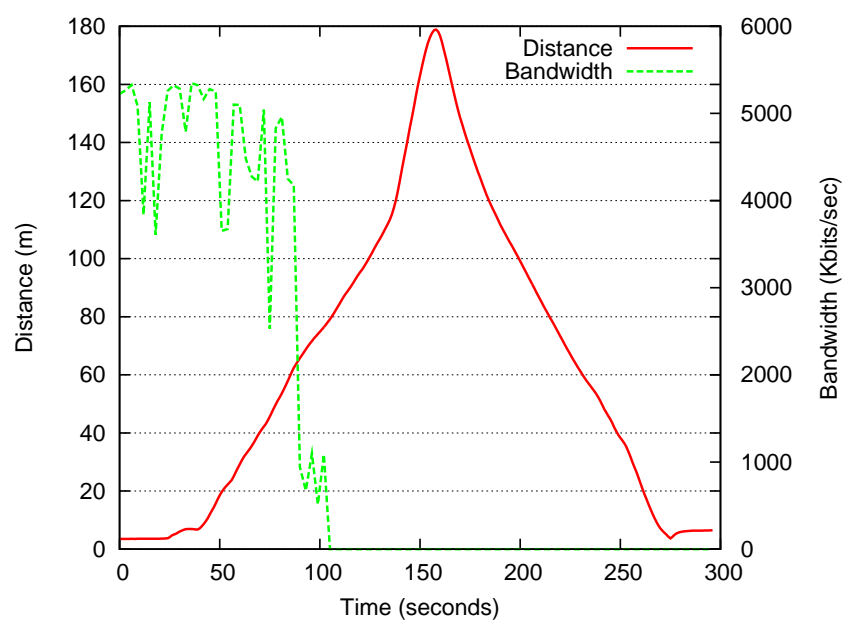

Figure 8: TCP range test with 2 cars (dist./band.).

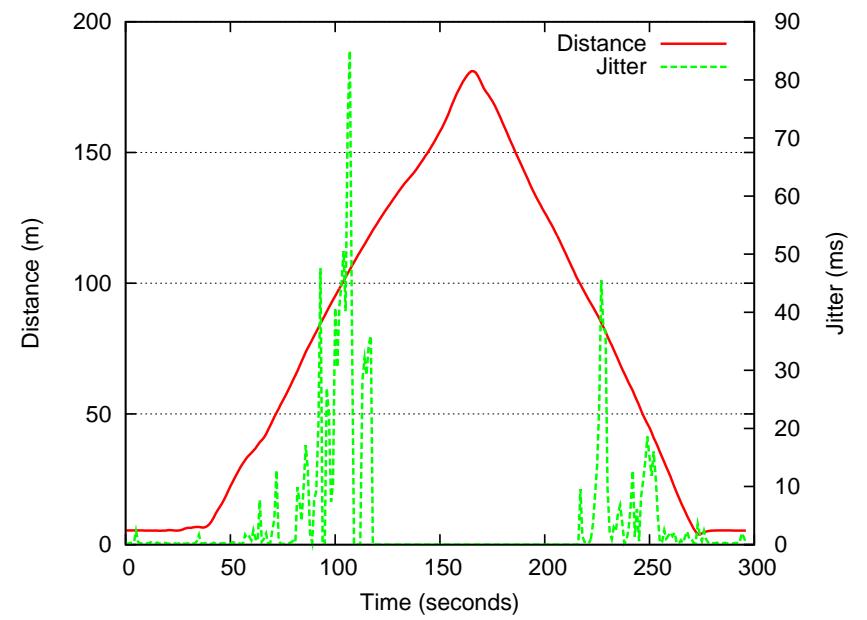

Figure 7: UDP range test with 2 cars (dist./jitter).

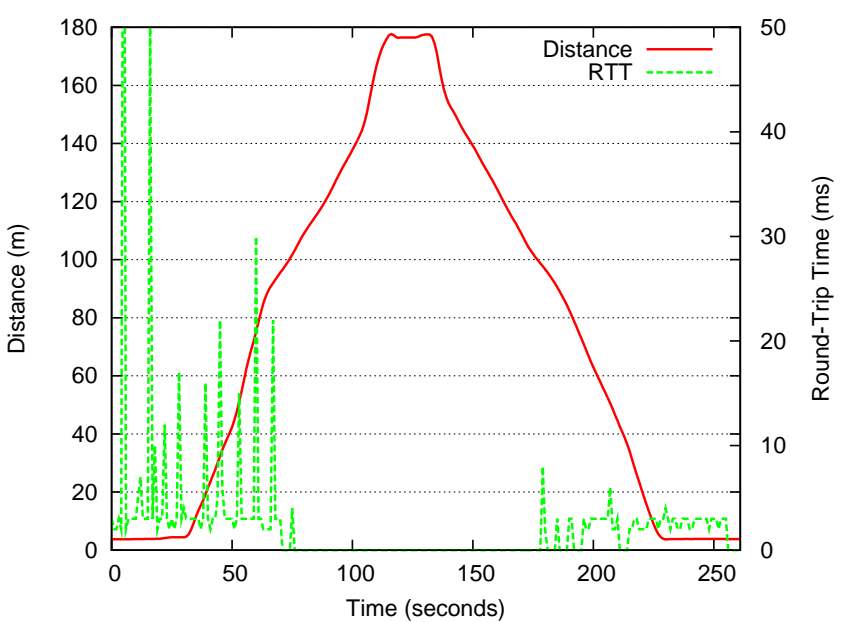

Figure 9: Ping range test with 2 cars (dist./RTT). 


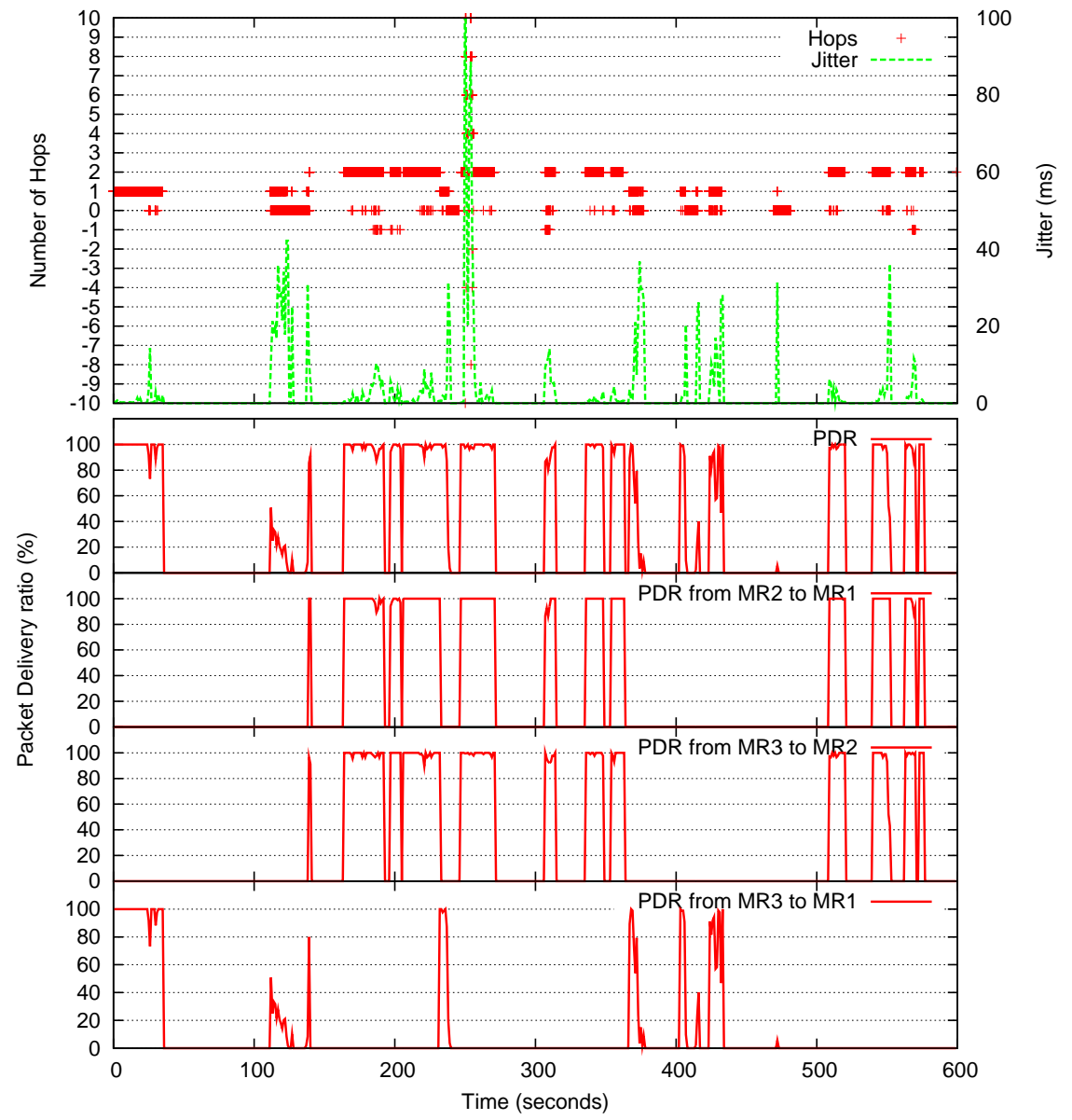

Figure 10: UDP urban test with 3 dynamic cars.
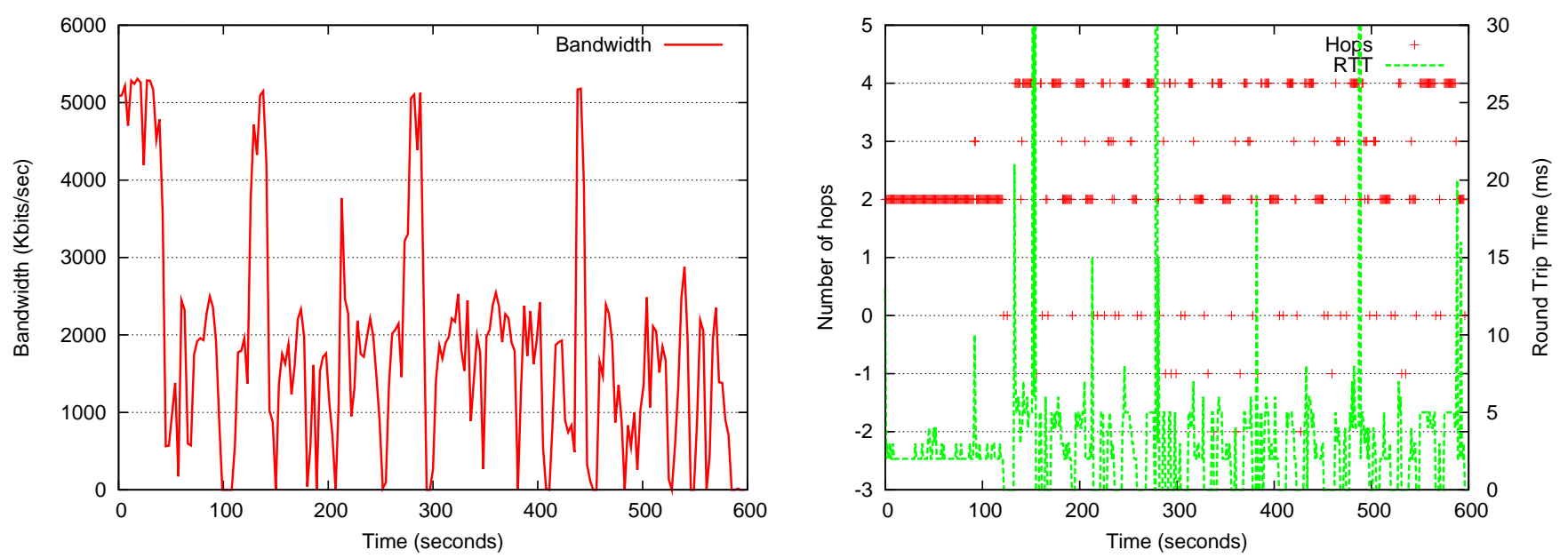

Figure 11: TCP urban test with 3 dynamic cars (band.). Figure 12: Ping urban test with 3 dynamic cars (hops/RTT). 


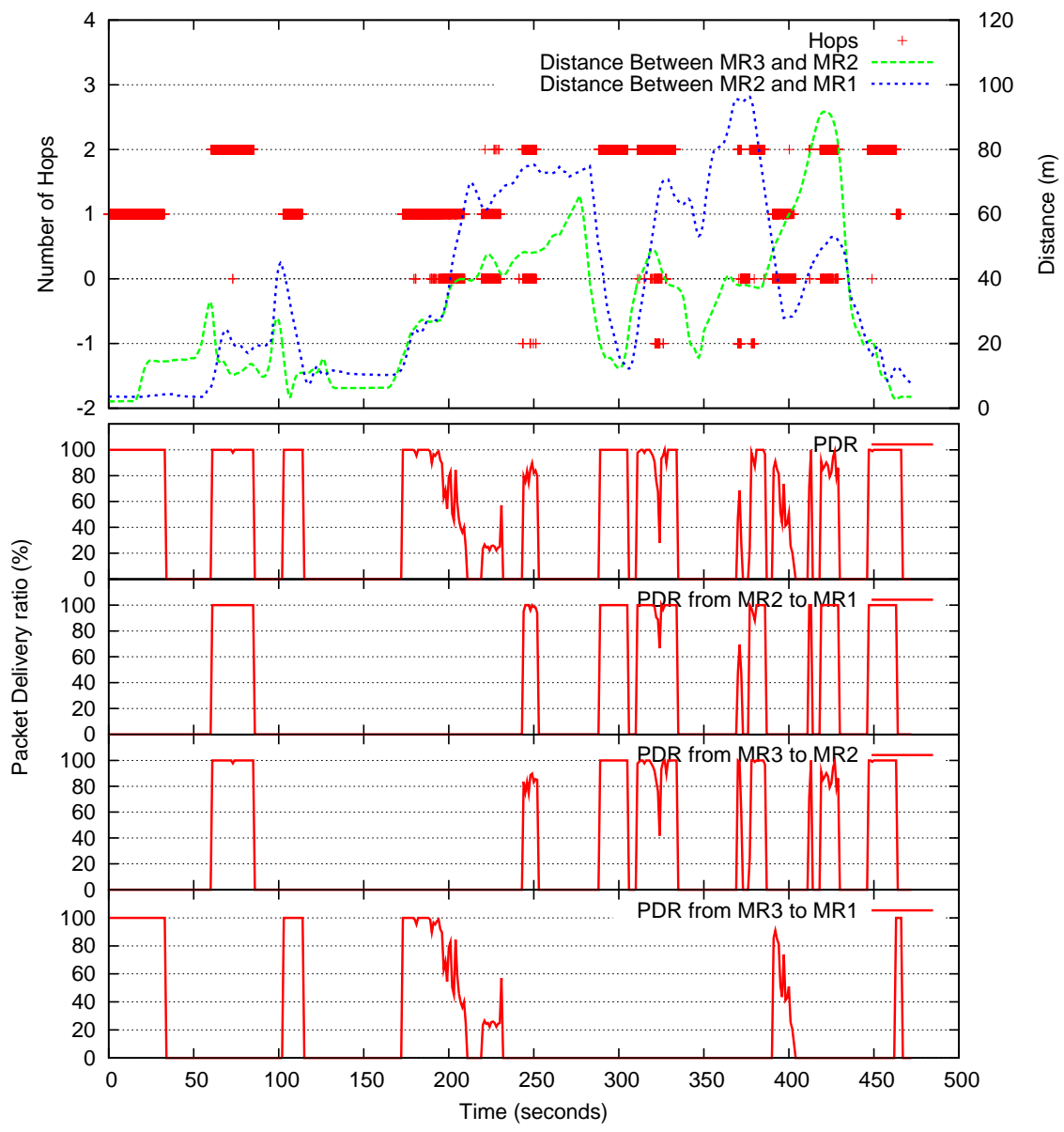

Figure 13: UDP highway test with 3 cars. 


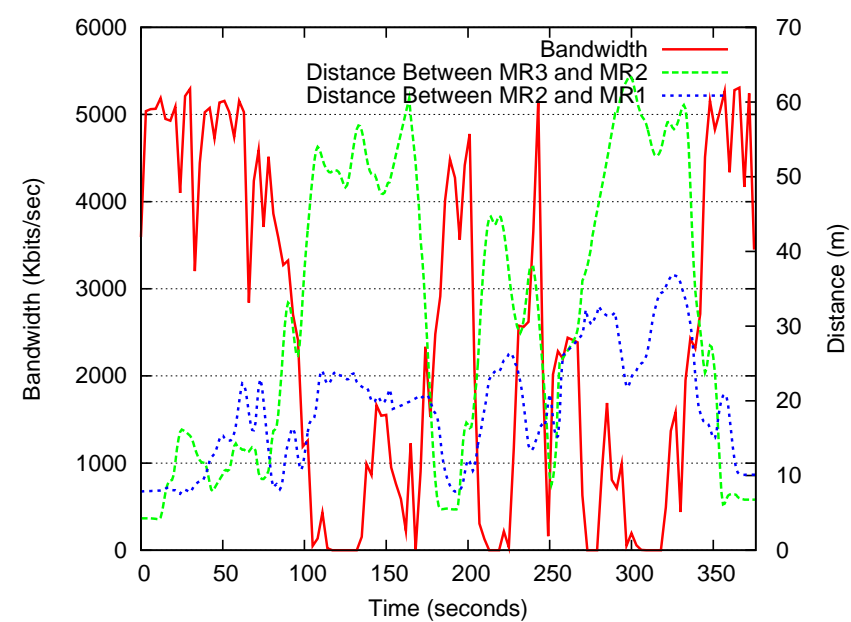

Figure 14: TCP highway test with 3 dynamic cars (band./dist.).

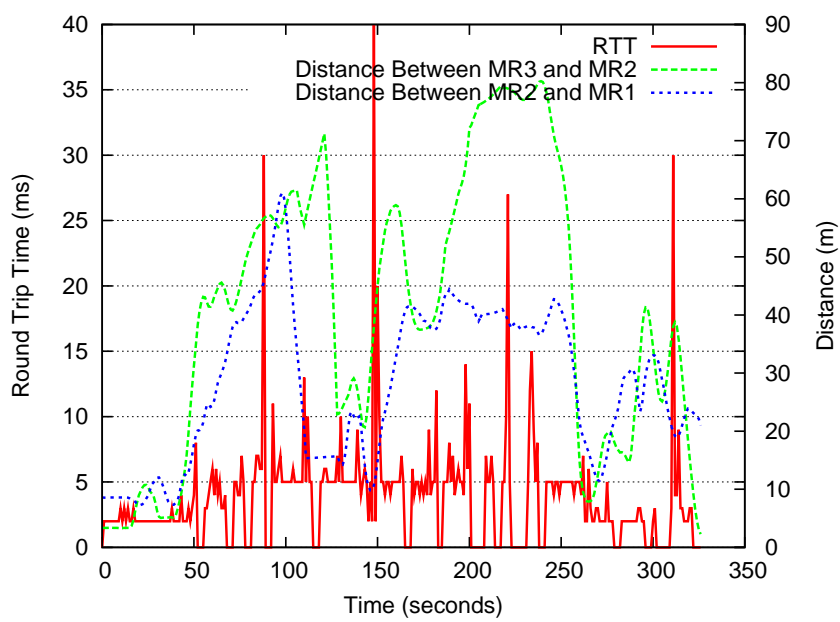

Figure 15: Ping highway test with 3 dynamic cars (RTT/dist.).

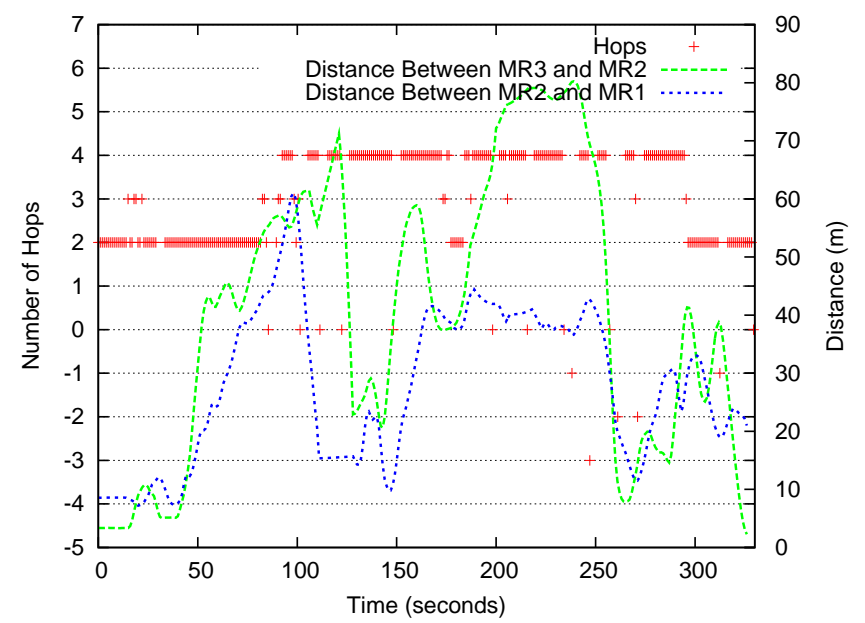

Figure 16: Ping highway test with 3 dynamic cars (hops/dist.). 

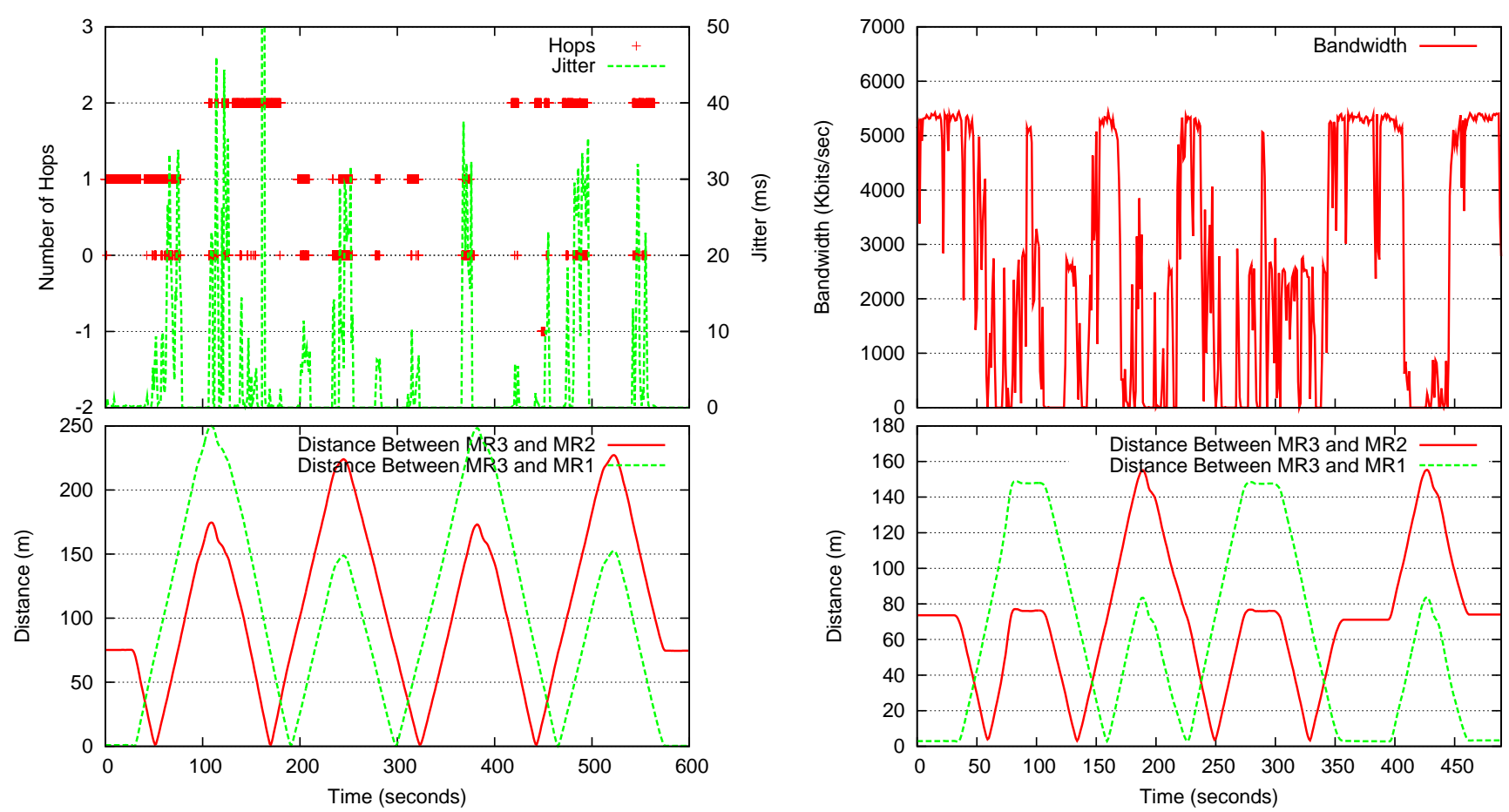

Figure 17: UDP overtaking test with 3 cars.

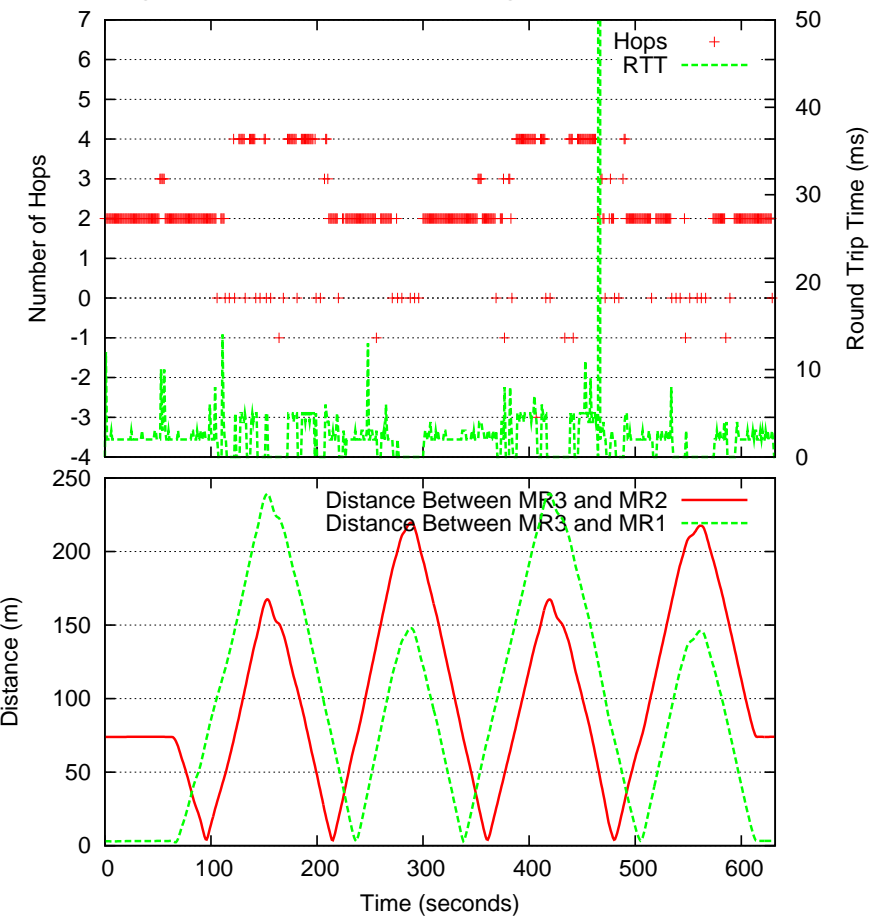

Figure 19: Ping overtaking test with 3 cars.

Figure 18: TCP overtaking test with 3 cars.

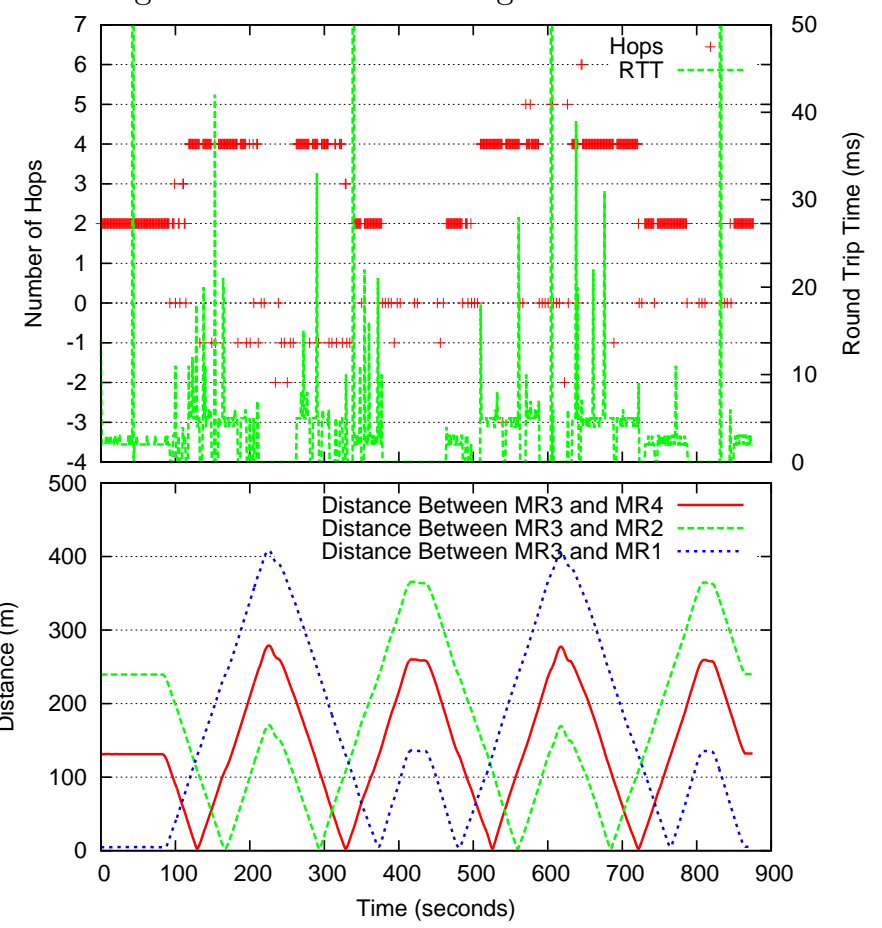

Figure 20: Ping overtaking test with 4 cars. 